Elaine Antonia de Paula ${ }^{a, b}$

José Tarcísio Buschinellib,d

Maria Maenoc

Roberto Fernandes da Costa ${ }^{e}$

${ }^{a}$ Centro de Referência em Saúde do Trabalhador Regional de Guarulhos. Guarulhos, SP, Brasil.

${ }^{\text {b} F u n d a c ̧ a ̃ o ~ J o r g e ~ D u p r a t ~ F i g u e i r e d o ~}$ de Segurança e Medicina do Trabalho. Programa de Pós-Graduação "Trabalho, Saúde e Ambiente”. São Paulo, SP, Brasil.

'Fundação Jorge Duprat Figueiredo de Segurança e Medicina do Trabalho. Coordenação de Saúde no Trabalho. Serviço de Medicina. São Paulo, SP, Brasil.

${ }^{d}$ Faculdade de Ciências Médicas da Santa Casa de São Paulo. São Paulo, SP, Brasil.

eFaculdade de Desenvolvimento do Rio Grande do Sul - FADERGS. Porto Alegre, RS, Brasil.

Contato:

Elaine Antonia de Paula

E-mail:

fisio.depaula@hotmail.com

Os autores declaram que o trabalho não foi subvencionado e que não há conflitos de interesses.

Trabalho baseado na dissertação de mestrado intitulada "Qualidade de vida de pacientes com afecções musculoesqueléticas relacionadas ao trabalho, atendidos no Centro de Referência em Saúde do Trabalhador do município de Guarulhos", defendida em 2013 no Programa de Pós-Graduação "Trabalho, Saúde e Ambiente" da Fundação Jorge Duprat Figueiredo de Segurança e Medicina do Trabalho - Fundacentro.

Trabalho apresentado na X Semana da Pesquisa da Fundacentro e posteriormente publicado na forma de resumo na página da instituição.

Recebido: 08/07/2015

Revisado: 18/05/2016

Aprovado: 25/05/2016

\section{Qualidade de vida de trabalhadores com LER/DORT e lombalgia ocupacional atendidos no Cerest de Guarulhos, São Paulo}

\author{
Quality of life of workers with RSI/WMSD and occupational low \\ back pain attended at the Workers' Health Reference Center of \\ Guarulhos, São Paulo, Brazil
}

\section{Resumo}

Objetivo: descrever a qualidade de vida (QV) dos trabalhadores com lesões por esforços repetitivos / distúrbios osteomusculares relacionados ao trabalho (LER/DORT) e lombalgia ocupacional (LO) atendidos no Centro de Referência em Saúde do Trabalhador Regional de Guarulhos, São Paulo, Brasil e verificar se fatores sociodemográficos, ocupacionais e relacionados ao quadro clínico interferem na sua QV. Métodos: estudo descritivo com delineamento transversal, utilizando-se o instrumento WHOQOL-Breve e uma entrevista estruturada. Dados colhidos entre 2011 e 2013. Resultados: participaram 192 sujeitos com LER/DORT e/ou LO. Os escores médios foram inferiores para todos os domínios do WHOQOL-Breve quando comparados à população saudável $(\mathrm{p}<0,001)$. Os trabalhadores com LER/DORT e LO simultaneamente apresentaram menores pontuações na QV geral e nos domínios físico, psicológico e ambiental. Aqueles que possuíam menor escolaridade apresentaram pontuação inferior na QV geral, e os que estavam afastados do trabalho, menores pontuações no domínio físico e na QV geral. Conclusão: as limitações do estudo permitem relacionar os resultados apenas com os sujeitos que efetivamente participaram dele. Todavia, os resultados encontrados corroboram várias pesquisas que têm constatado os efeitos deletérios dos distúrbios osteomusculares e de outras doenças crônicas na QV dos indivíduos afetados.

Palavras-chave: saúde do trabalhador; qualidade de vida; transtornos traumáticos cumulativos; lombalgia.

\begin{abstract}
Objective: to describe the quality of life (QOL) of workers with repetitive strain injury/work-related musculoskeletal disorders (RSI/WMSD) and/or occupational low back pain (LBP) attended at the Centro de Referência em Saúde do Trabalhador [Workers' Health Reference Center] of Guarulhos, São Paulo, Brazil, and to verify if sociodemographic, occupational and clinical factors interfere on their QOL. Methods: descriptive cross-sectional study using the World Health Organization Quality of Life (WHOQOL-Bref) instrument and a structured interview. Data collected between 2011 and 2013. Results: 192 individuals with RSI/WMSD and/or LBP participated. The average scores were lower for all WHOQOL-Bref domains when compared to the healthy population $(p<0,001)$. Workers who had simultaneously RSI/WMSDS and $L B P$ presented lower scores in the overall QOL and in physical, psychological and environmental domains. Workers with lower schooling had lower scores in the overall $Q O L$, and those who were on sick leave had lower scores in the physical domain and in the overall QOL. Conclusion: due to the limitations of this study its results can only be applied to the subjects who were engaged in it. However, the results corroborate various studies that have demonstrated the deleterious effects of musculoskeletal disorders and other chronic diseases on the affected individuals' QOL.
\end{abstract}

Keywords: occupational health; quality of life; cumulative trauma disorders; low back pain. 


\section{Introdução}

As afecções musculoesqueléticas que afetam os trabalhadores constituem um grande problema de saúde pública e um dos mais complexos na área da Saúde do Trabalhador (ST) ${ }^{1-5}$. Estudos mostram que esse grupo de agravos interfere na qualidade de vida (QV) dos trabalhadores acometidos na medida em que eles ficam limitados para realizarem suas atividades cotidianas, como o trabalho, o lazer, as atividades domésticas e de vida diária, além dos vários problemas que enfrentam no convívio social e familiar ${ }^{2-4,6-8}$.

Dessa forma, é importante que todos os profissionais envolvidos no tratamento e na reabilitação dos pacientes com lesões por esforços repetitivos/ distúrbios osteomusculares relacionados ao trabalho (LER/DORT) - nomenclatura oficialmente adotada no Brasil, pelos Ministérios da Saúde e Previdência Social - e lombalgia ocupacional (LO) adotem, em suas abordagens, o modelo biopsicossocial. Ele conduz a uma reabilitação profissional integrada e bem sucedida ao reconhecer que o processo saúde-doença e, consequentemente, a incapacidade gerada, decorre da interação das dimensões física, psicológica, biológica, sociocultural e ambiental em um todo dinâmico, em que cada qual tem seu significado e influencia a saúde e a QV do indivíduo, interagindo com as outras sem que haja uma dimensão mais importante ${ }^{9-13}$.

Com o intuito de enriquecer o escopo das pesquisas já existentes sobre as repercussões das LER/ DORT e LO na qualidade de vida dos trabalhadores, pretende-se, com este estudo, refletir a respeito da importância de uma avaliação abrangente que valorize a percepção do próprio trabalhador adoecido sobre sua condição atual, indo além da esfera física ao contemplar outros aspectos que são influenciados por esses agravos e podem levar à incapacidade para o trabalho. Acredita-se que a avaliação da QV possa trazer elementos relevantes que auxiliem os profissionais de saúde e todos os atores envolvidos na reabilitação desses trabalhadores a direcionarem seus esforços na adoção de estratégias que superem as condutas convencionais, como a prescrição de medicações e sessões de fisioterapia, buscando não apenas o alívio da dor, mas a "ressignificação" do trabalho, as potencialidades remanescentes e a melhora da QV e da capacidade funcional dos trabalhadores adoecidos, a fim de facilitar o retorno ao trabalho sustentado e contribuir para a implementação de políticas públicas e ações voltadas para o tratamento e a prevenção desse grupo de agravos. Assim, o objetivo do presente estudo foi descrever a qualidade de vida dos trabalhadores com LER/DORT e/ou LO atendidos no Centro de Referência Regional em Saúde do Trabalhador (Cerest) do município de
Guarulhos, bem como verificar se determinados fatores sociodemográficos, ocupacionais e relacionados ao quadro clínico interferem na QV apresentada por esses trabalhadores.

\section{Metodologia}

Trata-se de um estudo descritivo, com delineamento transversal e abordagem quantitativa, no qual se optou por considerar separadamente as LER/ DORT e a LO, a fim de englobar as lombalgias ocasionadas por eventos agudos e traumáticos, caracterizados como acidentes de trabalho, cujo mecanismo de produção da dor difere das LER/DORT, mas com sintomatologia e evolução clínica semelhantes ${ }^{5}$.

\section{População de estudo}

Para que a amostra fosse obtida, foram convidados os trabalhadores de ambos os sexos com diagnóstico de LER/DORT (com predomínio em membros superiores e coluna cervical, mas também em membros inferiores) e/ou LO (incluindo-se as lombalgias causadas por acidentes de trabalho) matriculados no Cerest Regional de Guarulhos no período de janeiro de 2011 a março de 2013. Considerando-se a similaridade da demanda de trabalhadores com LER/DORT e/ou LO atendidos no Cerest, com relação às características socioeconômicas, culturais, ocupacionais e clínicas, optou-se por uma amostra de conveniência, apesar de suas limitações. O intuito era o de conseguir um maior número de participantes, de acordo com a acessibilidade e a aceitação dos sujeitos para a participação na pesquisa, os quais foram convidados por meio de contato telefônico prévio. Esse tipo de amostragem tem sido frequentemente utilizado em pesquisas no campo da Saúde do Trabalhador ${ }^{14-16}$.

\section{Instrumentos}

Foi elaborado um roteiro de entrevista estruturada para fins desta pesquisa contendo questões sociodemográficas, ocupacionais e relacionadas ao quadro clínico apresentado pelos trabalhadores com LER/DORT e/ou LO.

Para avaliação da qualidade de vida, foi utilizado o questionário WHOQOL-Breve ${ }^{17}$, desenvolvido pela Organização Mundial da Saúde (OMS), cuja versão em português foi validada por Fleck et al. ${ }^{18}$. Esse instrumento de 26 questões possui duas questões gerais de qualidade de vida e as demais englobam os domínios físico, psicológico, social e ambiental.

O WHOQOL-Breve produz um perfil de QV que pode ser utilizado em levantamentos 
epidemiológicos. Fornece dados detalhados de uma população específica ou para a prática clínica ao permitir o reconhecimento dos aspectos que são mais afetados por uma doença e estabelecer escores basais nas áreas de domínio que engloba. Possibilita, também, observar mudanças na QV após intervenções ${ }^{17,18}$. Atualmente, o WHOQOL-Breve tem sido considerado um importante instrumento de avaliação da QV em diversas populações mundiais, incluindo trabalhadores de diferentes ocupações, sendo utilizado com frequência em pesquisas nacionais e internacionais ${ }^{19-21}$.

Este estudo foi aprovado pelo Comitê de Ética em Pesquisa da Faculdade de Saúde Pública da Universidade de São Paulo, com parecer n ${ }^{\circ}$ 257.522.

\section{Procedimentos e análise estatística}

Como parâmetro normativo para a comparação com os escores do WHOQOL-Breve obtidos pelos participantes da pesquisa, foram utilizados os valores apresentados no estudo de Cruz et al. ${ }^{22}$, cuja amostra foi composta pela população geral da região sul do Brasil, utilizada como referência para a população saudável do país.

Foram variáveis do estudo a qualidade de vida e seus domínios, os fatores sociodemográficos (faixa etária, gênero, escolaridade e renda mensal), a situação ocupacional e os aspectos relacionados ao quadro clínico (diagnóstico principal e presença de comorbidades crônicas associadas).

Com relação ao nível de escolaridade, optou-se por uma classificação simplificada para facilitar a comparação entre os grupos, dividindo-se os sujeitos em dois níveis educacionais: menor escolaridade (até o ensino fundamental completo, incluindo-se os sujeitos analfabetos) e maior escolaridade (a partir do ensino médio).

Em relação à situação ocupacional atual, realizou-se uma subdivisão, considerando-se os sujeitos que estavam trabalhando, aqueles que estavam afastados de suas atividades profissionais (incluindo-se os trabalhadores aposentados ou afastados pela previdência social) e os desempregados.

Quanto à renda mensal, realizou-se um agrupamento em duas categorias: com renda (incluindo os benefícios previdenciários por aposentadoria ou auxílio-doença) e sem renda.

Em relação às características clínicas dos trabalhadores adoecidos, a amostra foi dividida em três subgrupos, de acordo com o diagnóstico apresentado: 1) LER/DORT, acometendo membros superiores (MMSS), coluna cervical e/ou membros inferiores
(MMII); 2) lombalgia e 3) LER/DORT e lombalgia, simultaneamente.

Além disso, o relato de outras comorbidades crônicas associadas, como diabetes, hipertensão arterial sistêmica, bronquite crônica, entre outras, também foi investigado.

Os dados coletados foram analisados com auxílio do pacote computacional Statistical Package for the Social Sciences (SPSS), versão 18.0. Foi utilizado o teste não paramétrico de Komogorov-Smirnov para verificar o pressuposto de distribuição normal para cada variável do estudo. Os resultados foram apresentados por meio de estatística descritiva, expressos em média \pm desvio padrão e categorizados em porcentagem.

Para testar a hipótese de diferença entre as médias dos escores de cada domínio do WHOQOLBreve da amostra do presente estudo e a população geral, baseando-se nos valores obtidos pelo estudo de Cruz et al. ${ }^{22}$, foi utilizado o Teste t de Student para amostras independentes. O mesmo teste foi utilizado para as comparações em todas as variáveis que apresentavam dois grupos (gênero, escolaridade, renda mensal e comorbidades). Para as comparações entre grupos, naquelas variáveis que apresentavam mais do que dois grupos (faixa etária, situação ocupacional e diagnóstico), utilizou-se a ANOVA oneway seguida do teste de comparações múltiplas de Tukey. A comparação de proporções foi efetuada com o teste de qui-quadrado. Em todos os testes foi adotada significância estatística para $\alpha=0,05$.

\section{Resultados}

Do total de 394 trabalhadores com LER/DORT e/ ou LO matriculados no Cerest Regional de Guarulhos no período de janeiro de 2011 a março de 2013, 89 não puderam ser contatados por motivo de alterações cadastrais; 107 não puderam ou não consentiram participar da pesquisa; e 6 foram excluídos por apresentarem dificuldade de compreensão para responder aos questionários, o que resultaria em respostas imprecisas e pouco confiáveis. A amostra não probabilística foi, então, composta por 192 trabalhadores $(48,73 \%$ do total), com média de idade de 46,17 \pm 9,65 anos, ressaltando-se que o perfil dos respondentes em relação ao gênero, faixa etária e diagnóstico não diferiu dos não respondentes $(p>0,05)$.

As características sociodemográficas, ocupacionais e relacionadas ao quadro clínico são apresentadas na Tabela 1. 
Quanto às categorias profissionais dos participantes, as caracterizadas por demandas psicossociais e sobrecarga física de trabalho, como força, repetitividade e posturas inadequadas, representaram a quase totalidade da amostra. Destacaram-se as atividades de limpeza, como auxiliar de limpeza, empregada doméstica e diarista (15,6\%), trabalhadores da construção civil (13\%), ajudante geral (6,7\%), operador de máquina $(5,7 \%)$, auxiliar de linha de produção $(5,2 \%)$, costureira $(4,6 \%)$ e auxiliar de cozinha $(4,1 \%)$, dentre outras.

Na Tabela 2 é apresentada a distribuição dos participantes em relação à situação ocupacional separados por gênero, faixa etária e escolaridade. As mulheres representaram a maioria, entre os que estavam trabalhando $(63,4 \%)$ e os desempregados (60,6\%). Nos três cenários houve predominância dos trabalhadores entre 45 e 68 anos, sendo 64,5\% entre os que estavam trabalhando, 59,1\% entre os afastados e 57,6\% entre os desempregados.

Entre os sujeitos afastados de suas atividades profissionais ( $\mathrm{N}=66), 6$ já estavam aposentados (9\%), 38 estavam afastados há mais de um ano (57,5\%) e 22 há menos de um ano (33,3\%), dos quais apenas 7 trabalhadores $(11,6 \%)$ encontravam-se no programa de reabilitação profissional do INSS (Instituto Nacional do Seguro Social).

Tabela 1 Características sociodemográficas, ocupacionais e clínicas de trabalhadores com LER/ DORT $^{*}$ e/ou LO** matriculados no Cerest Regional de Guarulhos no período de janeiro de 2011 a março de 2013

\begin{tabular}{|c|c|c|}
\hline Variáveis & $N(192)$ & $\%$ \\
\hline \multicolumn{3}{|l|}{ Gênero } \\
\hline Feminino & 105 & 54,7 \\
\hline Masculino & 87 & 45,3 \\
\hline \multicolumn{3}{|l|}{ Faixa etária (anos) } \\
\hline 21 a 30 & 16 & 8,3 \\
\hline 31 a 44 & 58 & 30,2 \\
\hline 45 a 68 & 118 & 61,5 \\
\hline \multicolumn{3}{|l|}{ Escolaridade } \\
\hline Menor escolaridade (até o ensino fundamental completo) & 103 & 53,6 \\
\hline Maior escolaridade (a partir do ensino médio) & 89 & 46,4 \\
\hline \multicolumn{3}{|l|}{ Situação ocupacional durante a pesquisa } \\
\hline Trabalhando & 93 & 48,4 \\
\hline Afastado & 66 & 34,4 \\
\hline Desempregado & 33 & 17,2 \\
\hline \multicolumn{3}{|l|}{ Renda mensal } \\
\hline Com renda & 153 & 79,7 \\
\hline Sem renda & 39 & 20,3 \\
\hline \multicolumn{3}{|l|}{ Diagnóstico } \\
\hline LER/DORT & 77 & 40,1 \\
\hline LO & 50 & 26,0 \\
\hline LER/DORT + LO & 65 & 33,9 \\
\hline \multicolumn{3}{|l|}{ Comorbidade crônica } \\
\hline Sim & 47 & 24,5 \\
\hline Não & 145 & 75,5 \\
\hline
\end{tabular}

"LER/DORT: Lesões por esforços repetitivos / distúrbios osteomusculares relacionados ao trabalho

* LO: Lombalgia ocupacional 
Tabela 2 Trabalhadores com LER/DORT (lesões por esforços repetitivos/distúrbios osteomusculares relacionados ao trabalho) e/ou lombalgia ocupacional, segundo situação ocupacional, gênero, faixa etária e escolaridade, matriculados no Cerest Regional de Guarulhos no período de janeiro de 2011 a março de 2013

\begin{tabular}{|c|c|c|c|c|c|c|}
\hline \multirow[t]{2}{*}{ Variáveis } & \multicolumn{2}{|c|}{ Trabalhando } & \multicolumn{2}{|c|}{ Afastados } & \multicolumn{2}{|c|}{ Desempregados } \\
\hline & $N(93)$ & $\%(100)$ & $N(66)$ & $\%(100)$ & $N(33)$ & $\%(100)$ \\
\hline \multicolumn{7}{|l|}{ Gênero } \\
\hline Masculino & 34 & 36,6 & 40 & 60,6 & 13 & 39,4 \\
\hline Feminino & 59 & 63,4 & 26 & 39,4 & 20 & 60,6 \\
\hline \multicolumn{7}{|l|}{ Faixa etária (anos) } \\
\hline 21 a 30 & 8 & 8,6 & 5 & 7,6 & 4 & 12,1 \\
\hline 31 a 44 & 25 & 26,9 & 22 & 33,3 & 10 & 30,3 \\
\hline 45 a 68 & 60 & 64,5 & 39 & 59,1 & 19 & 57,6 \\
\hline \multicolumn{7}{|l|}{ Escolaridade } \\
\hline Menor escolaridade* & 54 & 58,0 & 32 & 48,5 & 17 & 51,5 \\
\hline Maior escolaridade ${ }^{\text {䊉 }}$ & 39 & 42,0 & 34 & 51,5 & 16 & 48,5 \\
\hline
\end{tabular}

*Até o ensino fundamental completo, incluindo-se os sujeitos analfabetos.

***: A partir do ensino médio.

Na Tabela 3 são apresentadas as médias e os desvios padrão dos escores do WHOQOL-Breve na população com LER/DORT e/ou LO em comparação aos valores normativos para os quatro domínios que compõem o instrumento, com base no estudo de Cruz et al. ${ }^{22}$.

De maneira geral, as médias de todos os domínios do WHOQOL-Breve na população com diagnóstico de LER/DORT e/ou LO foram significantemente menores do que os valores encontrados para a população saudável. As menores pontuações encontram-se nos domínios físico e ambiental.
A Tabela 4 mostra as médias e os desvios padrão dos escores de todos os domínios do WHOQOL-Breve e da qualidade de vida geral na amostra de trabalhadores com LER/DORT e/ou LO, estratificados por gênero, faixa etária, escolaridade, situação ocupacional atual, renda mensal, presença de comorbidades crônicas associadas e diagnóstico principal. Com relação ao diagnóstico, foram adotados os mesmos três subgrupos já mencionados: LER/DORT, lombalgia, LER/ DORT+lombalgia.

Tabela 3 Comparação entre as médias dos escores, segundo os domínios do WHOQOL-Breve, entre uma população saudável * e trabalhadores com LER/DORT* * e/ou LO*** matriculados no Cerest Regional de Guarulhos no período de janeiro de 2011 a março de 2013

\begin{tabular}{llcccc}
\hline \multicolumn{1}{c}{$\begin{array}{c}\text { Pacientes com } \\
\text { LER/DORT e/ou LO }\end{array}$} & \multicolumn{2}{c}{ População saudável } \\
Domínios & Média & $(\mathrm{n}=192)$ & Média & $(\mathrm{n}=751)$ & \\
\hline Físico & 46,05 & $(16,05)$ & 60,2 & $(9,4)$ & $\mathrm{p}<0,0001$ \\
Psicológico & 57,93 & $(15,78)$ & 65,9 & $(10,4)$ & $\mathrm{p}<0,0001$ \\
Social & 57,98 & $(21,23)$ & 75,8 & $(17,4)$ & $\mathrm{p}<0,0001$ \\
Ambiental & 43,57 & $(13,15)$ & 62,5 & $(14,2)$ & $\mathrm{p}<0,0001$ \\
\hline
\end{tabular}

*Valores normativos para os domínios do WHOQOL-Breve obtidos em uma amostra da população saudável da região sul do Brasil 22 .

糢LER/DORT: Lesões por esforços repetitivos / distúrbios osteomusculares relacionados ao trabalho.

橉粍LO: Lombalgia ocupacional. 
Tabela 4 Comparação dos valores médios dos estratos das variáveis estudadas, para cada um dos domínios do WHOQOL-Breve e para a QV geral entre trabalhadores com LER/DORT (lesões por esforços repetitivos/distúrbios osteomusculares relacionados ao trabalho) e/ou LO (lombalgia ocupacional) matriculados no Cerest Regional de Guarulhos no período de janeiro de 2011 a março de 2013

\begin{tabular}{|c|c|c|c|c|c|c|c|c|c|c|}
\hline \multirow{2}{*}{ Variáveis } & \multicolumn{2}{|l|}{ Físico } & \multicolumn{2}{|l|}{ Psicólogico } & \multicolumn{2}{|l|}{ Social } & \multicolumn{2}{|l|}{ Ambiental } & \multicolumn{2}{|l|}{ QV geral } \\
\hline & Média (DP) & Sig. & Média (DP) & Sig. & Média (DP) & Sig. & Média (DP) & Sig. & Média (DP) & Sig. \\
\hline \multicolumn{11}{|l|}{ Gênero } \\
\hline Feminino & $45,74(15,74)$ & \multirow{2}{*}{ NS } & $56,01(15,62)$ & \multirow{2}{*}{ NS } & $57,61(19,85)$ & \multirow{2}{*}{ NS } & $42,17(12,47)$ & \multirow{2}{*}{ NS } & $47,07(12,99)$ & \multirow{2}{*}{ NS } \\
\hline Masculino & $46,42(16,51)$ & & $60,24(15,75)$ & & $58,43(22,89)$ & & $45,26(13,82)$ & & $48,66(14,25)$ & \\
\hline \multicolumn{11}{|l|}{ Faixa etária (anos) } \\
\hline 21 a 30 & $46,87(14,03)$ & \multirow{3}{*}{ NS } & $65,11(16,22)$ & \multirow{3}{*}{ NS } & $64,58(22,46)$ & \multirow{3}{*}{ NS } & $45,31(12,07)$ & \multirow{3}{*}{ NS } & $45,31(12,07)$ & \multirow{3}{*}{ NS } \\
\hline 31 a 44 & $42,79(15,88)$ & & $55,53(17,08)$ & & $56,60(22,92)$ & & $41,75(11,70)$ & & $41,75(11,70)$ & \\
\hline 45 a 68 & $47,54(16,28)$ & & $58,08(18,32)$ & & $57,76(20,19)$ & & $44,22(13,95)$ & & $44,22(13,95)$ & \\
\hline \multicolumn{11}{|l|}{ Escolaridade } \\
\hline Menor escolaridade & $45,18(16,05)$ & \multirow{2}{*}{ NS } & $56,91(18,24)$ & \multirow{2}{*}{ NS } & $55,98(20,62)$ & \multirow{2}{*}{ NS } & $42,59(12,74)$ & \multirow{2}{*}{ NS } & $46,60(13,12)$ & \multirow{2}{*}{0,012} \\
\hline Maior escolaridade & $47,07(16,08)$ & & $59,03(17,47)$ & & $60,30(21,79)$ & & $44,69(13,60)$ & & $49,16(14,00)$ & \\
\hline \multicolumn{11}{|l|}{ Situação ocupacional atual } \\
\hline Trabalhando & $49,50(16,38)$ & $\mathrm{a}$ & $60,62(18,09)$ & \multirow{3}{*}{ NS } & $60,84(22,38)$ & \multirow{3}{*}{ NS } & $44,96(12,99)$ & \multirow{3}{*}{ NS } & $50,54(13,90)$ & A \\
\hline Afastado & $40,21(13,74)$ & $\mathrm{b}$ & $55,11(17,01)$ & & $53,91(19,84)$ & & $42,71(13,76)$ & & $44,12(12,05)$ & B \\
\hline Desempregado & $48,05(16,56)$ & $a b$ & $55,80(18,34)$ & & $58,08(19,82)$ & & $41,38(12,30)$ & & $47,34(14,05)$ & $\mathrm{Ab}$ \\
\hline \multicolumn{11}{|l|}{ Renda Mensal } \\
\hline Com renda & $46,45(16,06)$ & \multirow{2}{*}{ NS } & $58,71(17,86)$ & \multirow{2}{*}{ NS } & $58,66(22,06)$ & NS & $44,46(13,27)$ & IS & $48,51(13,52)$ & NS \\
\hline Sem renda & $44,50(16,11)$ & & $54,69(17,75)$ & & $55,34(17,57)$ & 然 & $40,06(12,24)$ & J & $44,97(13,52)$ & ivs \\
\hline Diagnóstico & & & & & & & & & & \\
\hline LER/DORT & $50,18(17,74)$ & $\mathrm{a}$ & $61,36(14,97)$ & $\mathrm{a}$ & $62,44(21,22)$ & & $46,71(14,21)$ & $\mathrm{a}$ & $52,02(14,22)$ & A \\
\hline LO & $44,49(14,10)$ & $a b$ & $57,08(16,71)$ & $a b$ & $56,00(21,09)$ & NS & $43,56(11,10)$ & $a b$ & $46,65(11,34)$ & $\mathrm{Ab}$ \\
\hline LER/DORT + LO & $42,36(14,38)$ & $\mathrm{b}$ & $54,52(15,39)$ & b & $54,23(20,68)$ & & $39,85(12,51)$ & $b$ & $43,65(13,05)$ & B \\
\hline Comorbidade & & & & & & & & & & \\
\hline Sim & $45,06(15,89)$ & NS & $54,16(13,73)$ & NS & $54,43(18,86)$ & NS & $43,81(13,50)$ & NS & $46,23(13,11)$ & NS \\
\hline Não & $46,37(16,14)$ & & $59,04(16,39)$ & Th & $59,13(21,87)$ & & $43,49(13,09)$ & & $48,29(13,71)$ & S \\
\hline
\end{tabular}

Obs.: Sig = significância; NS = não significante; Explicação do sistema de letras: um valor só é estatisticamente diferente de outro se não houver letras iguais. Assim, um valor identificado como "a" é diferente de um valor identificado com "b”, mas não de um valor identificado com "ab”.

Não foram encontradas diferenças significantes para as medidas de QV entre as variáveis gênero, faixa etária, renda mensal e presença de comorbidades crônicas associadas. Em relação à escolaridade, houve diferença somente no escore de QV geral, com média inferior para o grupo com menor escolaridade. Para a variável "situação ocupacional atual", diferenças significantes foram encontradas no domínio físico e na QV geral entre os sujeitos que estavam trabalhando e os afastados, com menor pontuação para os últimos.

Em relação ao diagnóstico, foram encontradas diferenças significantes na QV geral e nos domínios físico, psicológico e ambiental entre os sujeitos dos subgrupos 1 (LER/DORT) e 3 (LER/DORT e lombalgia). Os sujeitos acometidos, simultaneamente, por mais de um diagnóstico (terceiro subgrupo) apresentaram as menores médias nesses domínios e na QV geral.

\section{Discussão}

No presente estudo, as médias dos escores em todos os domínios do WHOQOL-Breve apresentadas pelos trabalhadores com LER/DORT e/ou LO foram menores que os considerados normais para a população saudável, baseando-se no estudo de referência realizado por Cruz et al. ${ }^{22}$. Esses resultados corroboram várias pesquisas que têm constatado os efeitos deletérios dos distúrbios osteomusculares e de outras doenças crônicas na QV dos indivíduos afetados $^{7,23-27}$. 
Segundo Roux et al. ${ }^{7}$, essa evidência de impacto negativo dos distúrbios musculoesqueléticos na QV reforça as recentes recomendações para o tratamento precoce e a prevenção desses agravos. Além disso, essas comparações são úteis por indicarem claramente as prioridades de recursos e intervenções e, ainda, por reforçarem a importância de iniciativas nacionais que priorizem essas áreas com base nos resultados de avaliações obtidos por meio dos relatos dos próprios pacientes ${ }^{27}$.

No presente estudo não foram encontradas diferenças significantes nas medidas de QV apresentadas pelos trabalhadores com LER/DORT e/ou LO em relação à presença de comorbidades crônicas, ressaltando-se que apenas uma pequena parcela da amostra $(24,5 \%)$ apresentava essa condição. Esse resultado contraria os achados de Salaffi, De Angelis e Grassi ${ }^{25}$, os quais constataram que a presença de outras comorbidades crônicas em pacientes com distúrbios musculoesqueléticos apresenta associação com as medidas de QV, exercendo maior impacto negativo sobre elas. Talvez, essa diferença possa ser explicada pela limitação da amostra não probabilística do presente estudo e/ou pela perda amostral.

Os trabalhadores com LER/DORT e lombalgia simultaneamente apresentaram menores pontuações na QV geral e nos domínios físico, psicológico e ambiental do WHOQOL-Breve, com diferença significante para o primeiro subgrupo, composto por trabalhadores que apresentavam somente LER/ DORT. Uma provável explicação seriam as condições mais sintomáticas e incapacitantes impostas pelo comprometimento simultâneo de vários segmentos corporais, como acontece com os trabalhadores que possuem ambos diagnósticos, causando limitação acentuada para o desempenho das atividades cotidianas, incluindo as laborativas e, consequentemente, exercendo maior influência negativa na QV. Esses resultados corroboram o estudo de Alonso et $\mathrm{al}^{28}$, cujos achados mostraram pontuações menores na QV para os indivíduos com condições de saúde crônicas quando comparados às pessoas saudáveis, principalmente na dimensão física, quando as doenças eram mais incapacitantes.

No que tange às variáveis gênero e idade, não foram encontradas diferenças nas medidas de QV, embora, de acordo com Roux et al. ${ }^{7}$, a combinação entre idade e gênero seja sempre necessária, pois os valores apresentados nas diferentes dimensões da QV, em geral, diminuem com o avançar da idade e são menores em mulheres do que em homens na fase adulta. De acordo com Yilmaz et al. ${ }^{26}$, de modo geral, é possível afirmar que de fato a QV piora com a idade, porém, essa tendência é mais evidente em relação às queixas álgicas pelo corpo, ao funcionamento físico e à saúde geral.No estudo de Skevington e McCrate ${ }^{27}$, os quais investigaram a relação de algumas variáveis sociodemográficas com a QV em pacientes com diversas condições de saúde utilizando o WHOQOL-Breve, as diferenças de faixa etária foram confirmadas em relação à influência na QV, a qual, em geral, foi melhor avaliada pelos sujeitos mais jovens que participaram da pesquisa. Em relação ao gênero, esses autores também confirmaram a influência dessa variável nas medidas de QV, indicando que as mulheres apresentaram pontuação superior apenas no domínio social em relação aos homens, mas inferior em todos os outros domínios do WHOQOL-Breve, principalmente no psicológico, o qual correspondeu à maior desigualdade de gênero.

Em nossa pesquisa, $61,4 \%$ da amostra encontrava-se na faixa etária de 45 a 68 anos, dos quais 13 trabalhadores (11\%) tinham 60 anos ou mais, refletindo o envelhecimento dessa população, já comprometida por uma doença relacionada ao trabalho. A literatura tem mostrado que a maioria dos trabalhadores apresenta uma redução na capacidade para o trabalho com o envelhecimento, cuja intensidade depende do contexto funcional e psicossocial desses trabalhadores ${ }^{6,29}$.

Cabe ressaltar que os novos paradigmas produtivos sustentam uma cultura organizacional nas empresas pouco receptiva aos trabalhadores mais velhos ${ }^{30}$. À velhice associam-se outros elementos que configuram a vulnerabilidade ocupacional (VO) caracterizada, segundo Fantini ${ }^{31}$, por baixa escolaridade e qualificação profissional, piores condições socioeconômicas, sexo feminino e trabalhadores não brancos. Esses autores constataram, em seu estudo, a associação entre VO e dor musculoesquelética quando os trabalhadores se encontravam em situações de altas demandas físicas e psíquicas, de baixo controle sobre o trabalho, apoio social insuficiente e piores condições de trabalho.

De um modo geral, a caracterização de nossa amostra revela essa VO por ser a maioria representada por mulheres, trabalhadores de meia idade e idosos, com baixa escolaridade, exercendo atividades menos qualificadas e com piores condições socioeconômicas e de trabalho, além do longo tempo de afastamento do trabalho devido a uma doença crônica, o que diminui sobremaneira as perspectivas para o futuro e contribui para uma pior percepção da QV, dificultando a reabilitação profissional e a reinserção no mercado de trabalho.

Foi encontrada, em nossa amostra, diferença significante no escore de QV geral em relação aos níveis de escolaridade, apresentando pontuação inferior no grupo com menor escolaridade. Isso corrobora a pesquisa de Salaffi et al. ${ }^{25}$, cujos resultados mostraram a influência negativa da baixa escolaridade na QV de pacientes com afecções osteomusculares, mas com efeito negativo mais marcante na dimensão física. $\mathrm{O}$ 
estudo de Skevington e McCrate ${ }^{27}$ também apontou que os participantes com baixo nível de escolaridade apresentaram QV mais pobre, com maior impacto no domínio físico e menor influência no meio ambiente. Fantini ${ }^{31}$ mostrou em sua pesquisa que os trabalhadores com menores níveis de escolaridade foram agrupados no perfil de maior vulnerabilidade ocupacional, apresentando pior percepção sobre a saúde, capacidade para o trabalho e QV.

De acordo Skevington e McCrate ${ }^{27}$, o impacto deletério que a baixa escolaridade exerce sobre a QV indica a necessidade de se diferenciar como a atenção à saúde é realizada em pacientes com diferentes níveis educacionais.

Em nossa pesquisa, os trabalhadores com LER/ DORT e/ou LO, quando comparados à população saudável, apresentaram valores inferiores para os domínios psicológico e social, corroborando diversas pesquisas que abordam as consequências de doenças crônicas sobre a saúde mental e os aspectos sociais da vida dos indivíduos acometidos ${ }^{25,32-36}$.

Segundo Harter et al. ${ }^{37}$, a ansiedade e a depressão são agravantes da dor musculoesquelética crônica, além de, normalmente, serem sintomas que ocorrem simultaneamente aos distúrbios musculoesqueléticos, causando um impacto negativo na QV das pessoas afetadas, bem como em sua percepção e resposta à dor, o que também prejudica a adesão aos tratamentos e até as possibilidades de se beneficiarem das intervenções propostas.

Com relação à situação ocupacional atual, houve diferença significante no domínio físico e no escore de QV geral entre os sujeitos que estavam trabalhando e aqueles que estavam afastados do trabalho, com menores pontuações para os últimos.

O afastamento do trabalho associado à ideologia de culpabilização, à perda de identidade social, à invisibilidade da doença, além de sentimentos de angústia, medo e desvalia contribuem para o agravamento do sofrimento causado pela dor física, pelas limitações funcionais, pela destruição de projetos de vida e pela imposição de uma situação completamente nova a ser elaborada ${ }^{38}$. Períodos prolongados de afastamento são considerados barreiras para o processo de retorno ao trabalho, principalmente, devido às repercussões psicossociais negativas para o trabalhador adoecido ${ }^{39,40}$. De acordo com Toldrá et al. ${ }^{41}$, compreender os prejuízos de períodos prolongados de afastamento do trabalho e buscar mecanismos para diminuir esta situação representam um grande desafio para os Cerest e serviços de reabilitação profissional.

Além do tempo de afastamento $(57,5 \%$ dos participantes desse estudo estavam afastados do trabalho há mais de um ano), chama atenção em nossa amostra o fato de apenas uma pequena parcela dos trabalhadores afastados estar inserida no programa de reabilitação profissional do INSS, considerando que a maioria foi representada por trabalhadores formais $(75 \%)$, o que revela a ineficácia dos programas de retorno ao trabalho desenvolvidos em nosso país. De acordo com Lima et al. ${ }^{13}$, na atual realidade do sistema previdenciário brasileiro, muitas vezes, além do longo período de afastamento, o trabalhador com limitações físicas ou psíquicas é devolvido ao mercado de trabalho desprovido de qualquer possibilidade de se manter de forma sustentável, o que reforça a sua desinserção social e prejudica, significativamente, a previdência, o Sistema Único de Saúde (SUS), a assistência social, as empresas e toda a sociedade.

Em relação ao domínio ambiental do WHOQOLBreve, a amostra do presente estudo também apresentou resultados inferiores aos da população saudável. Segundo Fantini ${ }^{31}$, os indivíduos mais expostos em suas ocupações também são os mais vulneráveis às precárias condições de vida e, assim, os fatores ambientais também precisam ser considerados na avaliação dos trabalhadores adoecidos por contribuírem na articulação da VO e dor musculoesquelética. A avaliação da QV pelo domínio ambiental representa mais uma medida de grande relevância para aplicações em saúde pública e outras iniciativas políticas e sociais, representando uma adição substancial ao conceito de $\mathrm{QV}^{27}$.

De acordo com Loisel et al. ${ }^{12}$, as causas da incapacidade não decorrem apenas das características individuais do sujeito (físicas e psicossociais), mas também se originam do meio ambiente em que ele está inserido, incluindo-se o seu local de trabalho, a previdência e o sistema de saúde. Nesse sentido, os programas de ST que tenham como foco o indivíduo e todas as suas dimensões são fundamentais, reafirmando a necessidade do desenvolvimento de um novo conceito nesse campo que extrapole o ambiente laboral ${ }^{21}$.

Cabe sinalizar que a ausência de um item no WHOQOL-Breve sobre aspectos do ambiente de trabalho demonstra uma limitação do instrumento, ao se reconhecer a importância e a centralidade do trabalho na vida do ser humano, representando um importante determinante social de saúde, cujas características, segundo Kudielka et al. ${ }^{42}$, podem representar um fator de risco independente para o desenvolvimento de doenças e contribuir para uma pobre QV.

Tendo em vista que se pretendia avaliar todos os trabalhadores com LER/DORT e/ou LO para verificar os fatores que podem interferir na QV, a amostragem não probabilística e a perda amostral de mais de 50\% podem ter limitado as inferências desejadas, sendo possível relacionar os resultados apenas com os sujeitos que efetivamente participaram do estudo. Assim, com a finalidade de superar algumas limitações 
importantes do presente estudo, como a perda amostral e o critério não probabilístico de amostragem, além das limitações inerentes aos estudos transversais, sugere-se a ampliação desta investigação, sobretudo no que se refere à capacidade de extrapolar os resultados encontrados para todo o universo de trabalhadores acometidos por esse grupo de agravos. De acordo com Roux et al. ${ }^{7}$, o padrão e a magnitude do efeito das afecções musculoesqueléticas na QV são melhor analisados longitudinalmente, em estudos de coorte, assegurando-se que os dados de referência sejam coletados antes do surgimento da condição.

Além disso, ao se analisar os valores de referência para a QV da população saudável do Brasil, propostos no estudo de Cruz et al. ${ }^{22}$, é importante destacar que as características socioeconômicas e culturais de sua amostra, pertencente à região Sul, podem ser bem distintas dos participantes do presente estudo, indicando a necessidade de pesquisas mais abrangentes que estabeleçam parâmetros normativos para a QV da população brasileira nas diferentes regiões do país, aumentando-se, assim, a sua força de evidência.

Considera-se também necessário complementar os estudos quantitativos com abordagens qualitativas que permitam a reflexão das características socioculturais próprias de cada país ou região, além dos diferentes valores e subjetividade de cada sujeito, informações nem sempre captadas com a aplicação de um único instrumento.

Vale ressaltar que, nesta pesquisa, ao se propor a utilização das informações obtidas por um instrumento de autoavaliação da QV, cujos resultados são expressos em escores, como o WHOQOL-Breve, pretende-se ir além de uma mera contabilização dos dados quantitativos coletados, buscando estimular o raciocínio clínico para outros aspectos, além dos físicos, que são afetados pelas LER/DORT e LO e que podem influenciar a incapacidade para o trabalho, baseando-se em um referencial teórico que valorize a perspectiva do próprio trabalhador e priorize o desenvolvimento de ações com a finalidade de reduzir o impacto da doença em sua qualidade de vida e favorecer o retorno sustentável ao trabalho.

Nesse contexto, as estratégias para a reabilitação e para os programas de retorno ao trabalho devem possibilitar aos profissionais e aos trabalhadores adoecidos reconhecer não só as limitações, mas principalmente as possibilidades físicas, psíquicas e sociais, incluindo a identificação das barreiras e dos facilitadores para o processo de reabilitação ${ }^{12,13,39-41}$. Assim, os profissionais devem orientá-los e estimulá-los a adotar uma postura ativa diante dos tratamentos propostos, proporcionando melhora da QV e mais rápida reintegração familiar e social ${ }^{5,12,32,38}$. É essencial que o plano de reabilitação contenha, como um dos elementos centrais, as ações de vigilância e as intervenções nos ambientes de trabalho, considerando-os como determinantes fundamentais para a gênese desses agravos ${ }^{5,12,39}$.

Acredita-se que este estudo nos leve a refletir sobre a importância de se buscar estratégias de enfrentamento para outros aspectos, além dos físicos, que possam influenciar a qualidade de vida dos trabalhadores com LER/DORT e LO e que interferem em sua reabilitação física, psicossocial e profissional.

\section{Contribuições de autoria}

Paula EA: elaboração do projeto e delineamento, levantamento de dados, elaboração do manuscrito, aprovação da versão final publicada; Buschinelli JT e Maeno M: contribuição substancial no projeto e delineamento, na sua análise e interpretação, contribuição importante na revisão crítica do manuscrito e aprovação da versão final publicada; Costa RF: contribuição substancial na análise e interpretação do projeto, realização das análises estatísticas, contribuição importante na revisão crítica do manuscrito e aprovação da versão final publicada.

\section{Referências}

1. Brasil. Secretaria de Políticas de Saúde. Protocolo de investigação, diagnóstico, tratamento e prevenção de Lesão por Esforços Repetitivos/ Distúrbios Osteomusculares Relacionados ao Trabalho. Brasília: Ministério da Saúde; 2000.

2. Merlo ARC et al. O trabalho entre prazer, sofrimento e adoecimento: a realidade dos portadores de lesões por esforços repetitivos. Psicol Soc. 2003;15:117-136.

3. Assunção AA, Almeida IM. Doenças osteomusculares relacionadas ao trabalho:
Membros superiores e pescoço. In: Mendes R. Patologia do trabalho. $2^{\mathrm{a}}$ ed. São Paulo: Atheneu; 2005. v. 2. p. 1501-1539.

4. Barbosa MSA, Santos RM,Trezza MCSF. A vida do trabalhador antes e após a Lesão por Esforço Repetitivo (LER) e Doença Osteomuscular Relacionada ao Trabalho (DORT). RevBrasEnferm.2007;60(5):491-6.

5. Maeno M,et al. Dor relacionada ao trabalho: lesões por esforços repetitivos (LER). Distúrbios osteomusculares relacionados ao trabalho 
(DORT). Protocolos de atenção integral à Saúde do Trabalhador de Complexidade Diferenciada. Brasília: Ministério da Saúde; 2012 [citado em 2013 mar 27]. Disponível em: http://bit.ly/2eFDI56

6. Walsh IAP, et al. Capacidade para trabalho em indivíduos com lesões musculoesqueléticas crônicas. Rev Saúde Pública.2004;38(2):149-56.

7. Roux CH,et al. Impact of musculoskeletal disorders on quality of life: an inception cohort study. Ann Rheum Dis.2005;64:606-11.

8. Schneider E,Irastorza X. OSH in figures: workrelated musculoskeletal disorders in the EU - facts and figures. Luxembourg: European Agency for Safety and Health at Work; 2010.

9. Minayo-Gomes C,Thedim-Costa SMSF. A construção do campo da saúde do trabalhador: percurso e dilemas. Cad Saúde Pública.1997;13Supl 2:S21-32.

10. Garbin AC, Neves IR, Batista RM.Etiologia do senso comum: as lesões por esforços repetitivos na visão dos portadores. CadPsicolSoc Trab.1998;1(1):43-55.

11. Trunchon M,Fillon L. Biopsychosocial determinants of chronic disability low back pain: a review. JOccupRehabil.2000;10:117-142.

12. LoiselP, et al.Disability prevention. New paradigm for the management of occupational back pain. DisManage Health Outcomes.2001;9(7):351-60.

13. Lima CQB, Tavares DS, Maeno M, organizadores. Proposta de diretrizes para uma política de reabilitação profissional. [S.l.]: Fundacentro; 2013.

14. Mendes LF,Lancman S. Reabilitação de pacientes com LER/DORT: contribuições da Fisioterapia em grupo. RevBras Saúde Ocup.2010;35(121):23-32.

15. Panzini RG,et al. Validação brasileira do instrumento de qualidade de vida/ espiritualidade, religião e crenças pessoais. RevSaudePublica.2011;45(1):153-65.

16. Caetano VC,et al. Processo saúde-doença: um estudo das representações sociais de trabalhadores com DORT. Physis.2012;22(3):1047-62.

17. Whoqol Group. Development of the World Health Organization WHOQOL-Bref quality of life assessment.Psychol Med.1998;28:551-8.

18. Fleck MPA,et al. Aplicação da versão em português do instrumento abreviado de avaliação da qualidade de vida. WHOQOL-bref. RevSaúdePública.2000;34(2):178-83.

19. Rusli BN,Edimansyah BA,Naing L. Working conditions, self-perceived stress, anxiety, depression and quality of life: a structural equation modelling approach. BMC Public Health. 2008;8(8) [citado em 2016 abr 28]. Disponível em: http://bit.ly/2eOIGu1

20. Fernandes MH, Rocha VM. Impact of the psychosocial aspects of work on the quality of life of teachers. Rev Bras Psiquiatria.2009;31(1):15-20.

21. Da Costa CSN,et al. Capacidade para o trabalho e qualidade de vida de trabalhadores industriais. Ciênc Saúde Coletiva.2012;17(16):1635-42.
22. Cruz LN,et al. Quality of life in Brazil: normative values for the Whoqol-bref in a southern general population sample. Qual Life Res.2011;20:1123-9.

23. Morken T, et al. Frequent musculoskeletal symptoms and reduced health-related quality of life among industrial workers. Occup Med (Lond).2002;52(2):91-8.

24. Picavet HSJ,Hoeymans N. Health related quality of life in multiple musculoskeletal diseases: SF-36 and EQ-5D in DMC3 study. Ann Rheum Dis.2004;63(6):723-9.

25. Salaffi F, De Angelis R, Grassi SW. Health-related quality of life in multiple musculoskeletal conditions: a cross-sectional population based epidemiological study. II. The MAPPING study. ClinExpRheumatol.2005;23(6):829-39.

26. Yilmaz F. Quality of life assessments with SF-36 in different musculoskeletal diseases. ClinRheumatol.2008;27:327-32.

27. Skevington SM,McCrate FM. Expecting a good quality of life in health: assessing people with diverse diseases and conditions using the WHOQOL-BREF. Blackwell Publishing Ltd Health Expectations.2011;15:49-62.

28. Alonso J, et al. Health-related quality of life associated with chronic conditions in eight countries: results from the International Quality of Life Assessment (IQOLA) Project. Qual Life Res. 2004;13:283-98.

29. Tuomi K, et al. Índice de capacidade para o trabalho. Helsinki: Instituto Finlandês de Saúde Ocupacional; 2005.

30. Peres MAC. O envelhecimento do trabalhador no contexto dos novos paradigmas organizacionais e os indicadores de exclusão por idade no trabalho. Linhas. 2005;6(2):1-23.

31. Fantini AJE. Dor musculoesquelética e vulnerabilidade ocupacional: uma abordagem dos perfis de acordo com o método GOM [dissertação]. Belo Horizonte: Universidade Federal de Minas Gerais; 2012.

32. Yeng LT, et al. Distúrbios osteomusculares relacionados ao trabalho. RevMed.2001;80(Ed. Esp. Pt. 2):422-42.

33. Bultmann U, et al. Health status, work limitations, and return-to-work trajectories in injured workers with musculoskeletal disorders. Qual Life Res.2007;16(7):1167-78.

34. Tuzun EH. Quality of life in chronic musculoskeletal pain.BaillieresBest PractRes, ClinRheumatol.2007;21(3):567-79.

35. Antonopoulou MD, et al.Studying the association between musculoskeletal disorders, quality of life and mental health. A primary care pilot study in rural Crete, Greece.BMC 
MusculoskeletDisord.2009;10(143) [citadoem 2013 nov 18]. Disponível em: http://bit.ly/2fy2wtC

36. Rocha NS, Fleck MP. Evaluation of quality of life in adults with chronic health conditions: the role of depressive symptoms. verBras Psiquiatr.2010;32(2):119-24.

37. Harter M, et al. Screening for anxiety. depressive and somatoform disorders in rehabilitationvalidity of HADS and GHQ-12 in patients with musculoskeletal disease. DisabilRehabil. 2001;23:737-44.

38. LimaA, Oliveira F. Abordagem psicossocial da LER: ideologia da culpabilização e grupos de qualidade de vida. In: Codo W, Almeida MCCG. LER: lesões por esforços repetitivos. Rio de Janeiro: Vozes; 1995. p.136-59.
39. Sato L. LER: objeto e pretexto para a construção do campo trabalho e saúde. Cad Saúde Pública. 2001;17(1):147-52.

40. Saldanha JHS,et al. Facilitadores e barreiras de retorno ao trabalho de trabalhadores acometidos por LER/DORT. RevBras Saúde Ocup.2013;38(127):122-38.

41. Toldrá RC,et al. Facilitadores e barreiras para o retorno ao trabalho: a experiência de trabalhadores atendidos em um Centro de Referência em Saúde do Trabalhador -SP, Brasil. RevBras Saúde Ocup.2010;35(121):10-22.

42. Kudielka BM,et al. Health-related quality of life measured by the SF12 in working populations: associations with psychosocial work characteristics. J Occup Health Psychol. 2005;10(4):429-40. 